\title{
Symptomatologie de la flore fongique infectant les feuilles d'accessions d'Arachis hypogaea L., (Fabaceae) provenant de Vavoua (centre-ouest) et Séguéla (nord-ouest) de la Côte d'Ivoire
}

\author{
${ }^{1}$ KOFFI Ahébé Marie Hélène ${ }^{*}{ }^{2}$ YAH N'Guéttia Marie, ${ }^{3}$ KOFFI N'Dodo Bony Clovis, ${ }^{4}$ KOFFI \\ Charlbert Yao, ${ }^{5}$ ATTA Taky Hortense Diallo \\ 1,2,3,4UFR Agroforesterie, Université Jean Lorongnon Guédé, Laboratoire d'Amélioration de Production Agricole, BP 150 \\ Daloa, Côte d'Ivoire \\ ${ }^{5}$ UFR Science de la Nature et de l'environnement, Université Nangui Abrogona, Laboratoire d'Amélioration de Production \\ Végétale, 02 BP 801 Abidjan 02, Côte d'Ivoire \\ *Auteur correspondant; E-mail : abebemarie77@yahoo.fr; Tel : (+225) 07489911
}

Mots clés: Arachides, symptômes, souches fongiques, Côte d'Ivoire.

Keywords: Peanuts, symptoms, fungal strains, Côte d'Ivoire.

Publication date 30/11/2020, http://m.elewa.org/Journals/about-japs /

1 RESUME

L'arachide est une culture d'intérêt pour les producteurs en Côte d'Ivoire. Elle s'insère bien dans les rotations de culture et correspond mieux aux types de sols dans plusieurs régions notamment le Nord et le Centre. Toutefois, sa production est limitée par certains genres fongiques qui causent des pertes quantitatives et qualitatives de production. Dans le but de contribuer à l'amélioration de la production de l'arachide en Côte d'Ivoire, une étude a permis d'isoler et d'identifier les genres fongiques associés aux feuilles malades des accessions d'arachide provenant de deux localités de la Côte d'Ivoire que sont Séguéla et Vavoua. Pour ce faire, un dispositif expérimental en blocs complètement randomisés de Fisher avec trois répétitions a été adopté. Chaque bloc a été constitué de six sous bloc espacés de $1 \mathrm{~m}$ les unes des autres avec 105 points de semis. Les taux de germination et d'infection des accessions ont été calculés et un inventaire des symptômes suivi d'une identification des différentes souches fongiques associées aux feuilles malades a été fait. L'analyse statistique a montré une différence significative au niveau des taux de germination (24 à 34\%) et d'infection (3,66 à 11 $\%$ ) des accessions d'arachide. Des feuilles malades, douze symptômes ont été observés et décrits dont quatre sont communs aux accessions des deux localités, deux uniquement à Séguéla et six à Vavoua. De ces symptômes, onze souches fongiques ont été identifiées à savoir les genres Aspergillus, Basipetospora, Colletotrichum, Curvularia, Fusarium, Geotrichum, Griseum Rhizoctonia, Pestalotiopsis, Pythium, Sclerotium et trois genres non identifiés. 


\begin{abstract}
Peanut is a crop of interest to producers in Côte d'Ivoire. It is well integrated into crop rotations and corresponds to soil types in several regions including the North and the Center. However, its production is limited by some fungal genera which causes quantitative and qualitative losses in production. In order to contribute to the improvement of peanut production in Côte d'Ivoire, a study was carried out to isolate and identify fungal genera associated with diseased leaves of peanut accessions from two localities in Côte d'Ivoire, Seguela and Vavoua. To do this, a completely randomized Fisher block experimental with three repetitions was adopted. Each block was composed of six sub-blocks spaced $1 \mathrm{~m}$ from each other with 105 seeding points. Germination and infection rates of accessions were calculated and an inventory of symptoms followed by identification of the different fungal strains associated with the diseased leaves was made. Statistical analysis showed a significant difference in the germination (24 to $34 \%)$ and infection (3.66 to $11 \%$ ) rates between peanut accessions. Of diseased leaves, twelve symptoms were observed and described, four of which are common to accessions of both localities, two only in Seguela and six in Vavoua. From these symptoms, eleven fungal strains were identified, these are Aspergillus, Basipetospora, Colletotrichum, Curvularia, Fusarium, Geotrichum, Griseum Rhizoctonia, Pestalotiopsis, Pythium, Sclerotium and three unidentified genera.
\end{abstract}

\section{INTRODUCTION}

L'Arachis bypogaea L. (Fabaceae) est une importante légumineuse à graine cultivées dans les régions tropicales, subtropicales et tempérées chaudes (Sharma et al., 2006; Guchi et al., 2014). Elle est la 13ème culture vivrière et la 4ème culture oléagineuse la plus importante au niveau mondial (Reddy et al., 2011). La production mondiale d'arachide non décortiquée est estimée à 45 millions de tonnes ( $90 \%$ ), avec plus de 67 $\%$ de cette production provenant d'Asie et $26 \%$ d'Afrique. En Afrique, l'arachide compte parmi les principales cultures d'exportation de plusieurs pays tels que la Guinée, le Soudan et le Ghana (Ndjeunga et al., 2006 ; Dzomeku et al., 2009. En Côte d'Ivoire, l'arachide est produite principalement dans les régions Nord et Centre du pays (Anader, 2009). Cette production a été estimée à 88000 tonnes (Faostat, 2013) et destinée à la consommation domestique ainsi que pour le commerce (Christie et al., 2015). Cependant, la majeur partie de cette production est consommée sur place et principalement sous forme de pâte d'arachide (Boli et al., 2013; Diakité et al., 2017). L'arachide contient 48-50\% de corps gras, 26-28\% de protéines et est riche en fibres minéraux et en vitamines (Noba, 2014). Malgré cette importance, la culture de l'arachide est confrontée à de nombreuses maladies causées par diverses moisissures phytopathogènes telle que la fonte des semis, la cercosporiose et la pourriture sèche des racines et des gousses qui réduisent le rendement et la qualité des graines tout en augmentant le coût de production (Yussif et al., 2014). De plus, outre les maladies en champ les graines d'arachide en conservation sont souvent contaminées par des moisissures du genre Aspergillus responsables de la production de mycotoxines qui entraînent d'énormes pertes pendant la germination (Ouattara et al., 2011; Guchi, 2015). En effet, les champignons représentent, en agriculture les principaux microorganismes responsables des pertes. Ainsi, $83 \%$ des maladies des plantes sont dues aux champignons et $17 \%$ provoquées par les bactéries et les virus (Boussaber et al., 2012). La FAO estime qu'au moins $25 \%$ de la récolte mondiales des denrées agricoles est contaminée par les mycotoxines (Manfred et Nicole, 2000) qui entrainent des pertes économiques importantes et des risques pour la santé publique, due à leur présence dans les aliments (Morgavi et Riley, 2007).

En Côte d'Ivoire, très peu d'études sur les champignons infectant les feuilles de l'arachide 
ont été effectuées. La connaissance précise de ces champignons pourrait permettre alors d'envisager des méthodes de lutte pendant la culture au champ. C'est alors, dans cette optique qu'une étude portant sur les différents champignons associés aux feuilles des accessions

\section{MATERIELET METHODES}

3.1 Site d'étude : L'étude a été réalisée sur le site de l'Université Jean Lorougnon Guédé située dans le département de Daloa en Côte d'Ivoire. La ville de Daloa est située dans la région du Haut Sassandra, au Centre-Ouest de la Côte d'Ivoire entre le $6^{\circ}$ et $7^{\circ}$ de latitude Nord et le $7^{\circ}$ et $8^{\circ}$ de longitude Ouest. Le substrat pédologique de Daloa appartient au vieux socle précambrien composé de granites, migmatites. Ces sols, lessivés et profonds $(20 \mathrm{~m})$ sont dus aux précipitations abondantes et à l'altération rapide des roches. Les sols de la région sont
d'Arachis hypogae provenant de deux localités (Vavoua et Séguéla) de la Côte d'Ivoire a été effectuée. L'objectif de cette étude est de caractériser les genres fongiques associés aux feuilles de quelques accessions d'Arachis hypogaea afin de minimiser les pertes de rendement.

majoritairement ferralitiques. Ils sont généralement très profonds avec un taux élevé de matière organique.

3.2 Matériel : Le matériel végétal utilisé dans cette étude a été constitué de graines apparemment saines et de feuilles infectées de six accessions d'Arachis hypogae. Les graines toutes courtes de ces accessions ont moins d'un an et proviennent des stocks de deux localités, Séguéla et Vavoua (Figure 1). Les feuilles infectées ont été prélevés sur les plants âgés d'un mois et demi de culture.

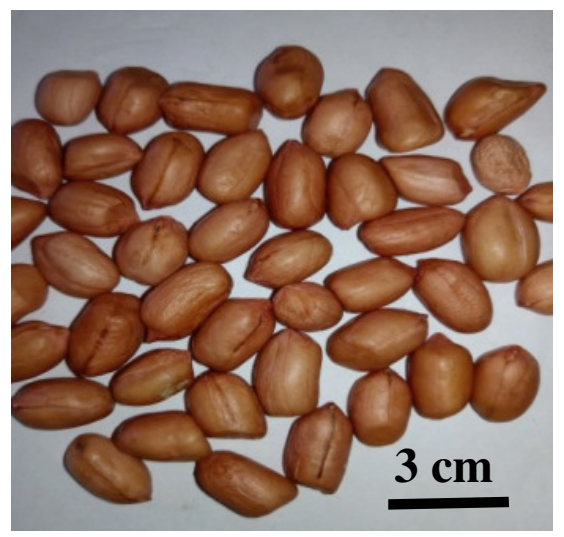

Figure 1 : Graine d'accessions d'Arachis hypogaea

\subsection{METHODES}

3.3.1 Dispositif expérimental : Le dispositif expérimental utilisé a été constitué d'un bloc de Fischer complètement randomisés avec trois répétitions. Une parcelle de $143 \mathrm{~m}^{2}(13 \mathrm{~m}$ x 11 $\mathrm{m})$ constituées de trois blocs a été mise en place. Les bordures de la parcelle ont été de $1 \mathrm{~m}$ et l'espacement entre deux blocs voisins de $2 \mathrm{~m}$. Chaque bloc a été constitué de six sous bloc espacés de $1 \mathrm{~m}$ les unes des autres. Chaque sous bloc a mesuré $1,5 \mathrm{~m}^{2}(1 \mathrm{~m} \times 1,5 \mathrm{~m})$ et a été constitué de 35 points de semi à raison de 3 graines par poquet avec 7 lignes soit 105 points de semis pour une seule accession. Au total 1890 points de semi ont été obtenus. Les semis ont été effectués de septembre à novembre 2019 correspondant à la petite saison des pluies. Après semis, un arrosage journalier et des désherbages si nécessaires ont été fait pendant la durée de la culture. Les essais ont été effectués sans aucune fertilisation des parcelles.

3.3.2 Calcul du taux de germination: Le taux de germination de chaque accession a été déterminé après apparition de l'hypocotyle à la surface du sol. A cet effet, quinze jours après semis les plants des différentes accessions ayant 
germés ont été comptés. Le taux de germination de chaque accession a été calculé par le rapport du nombre de graines ayant germé sur le nombre total de point de semi selon la formule cidessous.

$$
T G=\frac{\mathrm{Ngg}}{\mathrm{Ntgs}} \times 100
$$

TG (\%): Taux de germination

$\mathrm{Ngg}$ : Nombre de graines de l'accession ayant germé

Ntgs : Nombre total de graines semées par accession Ntgs $=35$

3.3.3 Calcul du taux d'infection : Un mois et demi après germination, le nombre de plants infectés pour chaque accession a été dénombré et le taux d'infection a été calculés comme ci :

$$
T I=\frac{\mathrm{Npi}}{\mathrm{Ntp}} \times 100
$$

TI (\%): Taux d'infection

Npi : Nombre de plant infecté

Ntp : Nombre total de plant pour une accession, avec Ntp $=105$

\subsubsection{Description des symptômes} observés : Les observations sur les feuilles des plants en culture ont été faites 1 mois et demi après semis. Les symptômes foliaires ont été observés et décrits pour chaque accession. A cet effet, l'aspect, la forme, la taille et la coloration des symptômes ont été décrits par observation visuelle et au touché. Les feuilles des accessions présentant les différents types de symptômes d'attaques fongiques ont été prélevées de manière aléatoire. Ainsi, cinq feuilles avec le même type de symptômes ont été collectées sur cinq plants et placées dans du papier buvard, puis dans un sachet plastique en polyéthylène pour les analyses au laboratoire.

3.3.5 Désinfection et purification des échantillons : Les feuilles d'arachide présentant les symptômes ont été découpées en explants de $2 \mathrm{~cm}$ sur le front de croissance des symptômes. Ces différents explants ont été désinfectés avec de l'hypochlorite de sodium $\left(8^{\circ} \mathrm{chl}\right)$ dilué à $10 \%$ pendant 3 min. Trois rinçages successifs de 3 min chacun a été fait avec de l'eau distillée stérile. Après élimination de l'excès d'eau sur du papier buvard stérile, sous une hotte, trois à quatre explants de feuilles ont été ensemencés dans des boîtes de Pétrie contenant du milieu pomme de terre enrichit au glucose (PDA). Ces boittes ont été par la suite scellées avec du papier film pour éviter toutes contaminations extérieures puis incubées dans une salle à la température ambiante $\left(25^{\circ} \mathrm{C}\right)$. Une ou deux purifications ont été nécessaires sur de nouveaux milieux PDA avant l'obtention de la souche pure d'un champignon.

3.3.6 Identification et caractérisation des souches fongiques: Deux semaines après culture sur le milieu PDA, les souches fongiques obtenues ont été observées à l'œil nu, afin de déterminer la coloration des colonies de champignons. Les différentes souches fongiques ont été par la suite observées au microscope à écran pour leur identification. Cette identification a été effectuée en utilisant les clés d'identification de Barnett et Hunter (1972) et de Botton et al. (1990). Les champignons identifiés ou non ont été décrits. Les observations ont porté sur des caractéristiques telles la couleur et la forme des spores, le cloisonnement et la ramification ou non de mycélium. Les isolats ont ensuite été regroupés en différents genres fongiques.

3.4 Analyses statistiques des données : Une analyse à un critère de classification 
(ANOVA 1) a été effectuée au seuil $\alpha=0,05$. Le logiciel Statistica 7.1 a été utilisé pour comparer les taux de germination et d'infection des différentes accessions. Un test LSD de Fisher a

\section{RESULTATS}

\subsection{Taux de germination, d'infection et de} mortalité

4.1.1 Taux de germination: L'analyse statistique des taux de germination a montré une différence entre les moyennes des différentes accessions d'arachide. Ces moyennes ont été faibles dans l'ensemble et ont variées de 24 à 34 $\%$. Deux groupes d'homogénéité ont été obtenus. Le $1^{\text {er }}$ groupe comprenant les accessions V1, V3, S2, S3 avec les taux de germination les plus élevés (31 à $34 \%$ ) et le $2^{\text {ème }}$ groupe, V2 et S1 avec les faibles taux de germination respectifs de 24,33 à $25 \%$ (Tableau 1). complété les analyses, quand une différence significative a été notée entre les valeurs pour le classement des moyennes.

4.1.2 Taux d'infection: Les résultats de l'analyse statistique ont montré que les taux d'infection diffèrent d'une accession à un autre $(\mathrm{P}=0,003)$. En effet, les accessions qui présentent les plus important taux d'infection sont les accessions S2 de Séguéla et V3 de Vavoua appartenant au premier groupe avec des taux d'infection respectifs de 9,66 à $11 \%$. Le second groupe d'homogénéité renferme les accessions à faible taux d'infection. Ce sont les accessions S1, V1, S3 et V2 avec les taux d'infections respectifs de 3,66, 5,00, 5,33 et $6 \%$ (Tableau 2).

Tableau 1 : Taux de germination ( \pm Ecart-type) des accessions d'arachides

\begin{tabular}{c|c|c|c}
\hline Accessions & Taux de germination ( \pm Ecart-type) & \multicolumn{2}{|c}{ Statistiques } \\
\cline { 3 - 4 } & & $\mathbf{F}$ & $\mathbf{P}$ \\
\hline V1 & $31,33 \pm 2,08^{\mathrm{a}}$ & & \\
V2 & $24,33 \pm 2,88^{\mathrm{b}}$ & 5,46 & 0,007 \\
V3 & $34,00 \pm 1^{\mathrm{a}}$ & & \\
S1 & $25,00 \pm 5,56^{\mathrm{b}}$ & & \\
S2 & $31,66 \pm 2,30^{\mathrm{a}}$ & & \\
S3 & $31,00 \pm 1^{\mathrm{a}}$ & & \\
\hline
\end{tabular}

V1, V2 et V3 : Accessions (1, 2 et 3) de Vavoua ;

S1, S2 et S3: Accessions (1, 2 et 3) de Séguéla

F : valeurs de Fischer, P : Probabilité. Les moyennes affectées de la même lettre dans une colonne sont statistiquement égales au seuil de $5 \%$ selon le test LSD de Fischer

Tableau 2 : Taux d'infection ( \pm Ecart-type) des accessions d'arachides

\begin{tabular}{c|c|c|c}
\hline Accessions & Taux d'infection $( \pm$ Ecart-type) & \multicolumn{2}{|c}{ Statistiques } \\
\cline { 3 - 4 } & & F & $\mathbf{P}$ \\
\hline V1 & $5,00 \pm 1^{\mathrm{b}}$ & & \\
V2 & $6,00 \pm 3^{\mathrm{b}}$ & 6,52 & 0,003 \\
V3 & $11,00 \pm 1^{\mathrm{a}}$ & & \\
S1 & $3,66 \pm 1,52^{\mathrm{b}}$ & & \\
S2 & $9,66 \pm 2,30^{\mathrm{a}}$ & & \\
S3 & $5,33 \pm 2,08^{\mathrm{b}}$ & & \\
\hline
\end{tabular}

V1, V2 et V3: Accessions (1, 2 et 3) de Vavoua;

S1, S2 et S3 : Accessions (1, 2 et 3) de Séguéla

$\mathrm{F}$ : valeurs de Fischer, P : Probabilité. Les moyennes affectées de la même lettre dans une colonne sont statistiquement égales au seuil de $5 \%$ selon le test LSD de Fischer 
4.2 Symptômes communs observés et champignons isolés des feuilles des accessions d'arachide de Vavoua et Séguéla : Quatre symptômes ont été observés sur les feuilles des accessions provenant des deux localités (Tableau 3). Le $1^{\mathrm{er}}$ symptôme est une tache de coloration brune au contour jaune sur la face supérieure de la feuille. De ce symptôme, deux champignons ont été isolés. Celui isolé des feuilles des accessions provenant de Séguéla présente une colonie noire sur le milieu PDA. L'observation microscopique a montré des mycéliums cloisonnés et ramifiés de Rhizoctonia sp. Par contre l'autre champignon isolé des feuilles des accessions provenant de Vavoua montre une colonie brune claire. L'observation microscopique a montré des mycéliums cloisonnés et ramifiés mais le champignon n'a pas pu être identifié (NI 1). Le $2^{\text {ème }}$ symptôme est une tache brune ronde au contour noir, avec un intérieur blanchâtre sur la face supérieure de la feuille. De ce symptôme, un seul champignon a été isolé sur les feuilles des accessions quelle que soit la localité de provenance. Ce champignon est blanc tapissé sur le milieu de culture. Au microscope, le champignon observé a montré un mycélium non cloisonné, ramifié et stérile de Sclerotium sp. Le $3^{\text {ème }}$ symptôme se caractérise par une tache noire entourée d'un halo jaune à la face supérieure de la feuille. Trois champignons ont été isolés à partir de ce symptôme. Les deux premiers non identifiés (NI 2 et NI 3) ont été isolés des feuilles des accessions provenant de Séguéla. Le champignon NI 2 est cotonneux de coloration grise sur le milieu PDA et l'aspect microscopique a montré des mycéliums non cloisonnés et ramifiés. Le deuxième champignon, NI 3 est blanc filamenteux sur milieu PDA. L'observation microscopique a montré des mycéliums cloisonnés non ramifiés. Le troisième champignon a été isolé des feuilles des accessions provenant de Vavoua. Il est marron floconneux sur milieu PDA. L'observation microscopique a montré des mycéliums cloisonnés et ramifiés de Pythium sp. Le $4^{\text {ème }}$ symptôme se présente sous formes de taches noires sur les bordures des feuilles. De ce symptôme, deux champignons ont été isolés et identifiés sur les feuilles des accessions provenant de Vavoua et il en est de même pour les feuilles des accessions provenant de Séguéla: Des feuilles provenant de Séguéla, Curvularia sp. d'aspect strié rose blanc et Colletotrichum sp. de coloration orangée dispersée dans le milieu PDA ont été isolés. Egalement, des feuilles provenant de Vavoua, deux autres genres, Fusarium sp. d'aspect cotonneux de couleur grise et Pestalotiopsis sp. d'aspect cotonneux et brun sombre sur le milieu PDA ont été isolés. 
Tableau 3 : Caractéristiques macroscopiques et microscopiques des souches fongiques isolées des symptômes communs aux feuilles des accessions provenant de Vavoua de Séguéla

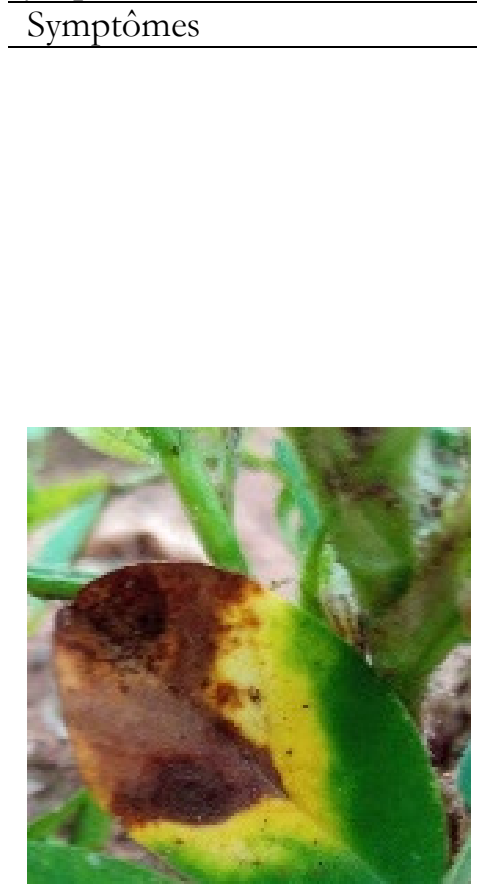

Coloration brune au contour jaune

Localités Aspect macroscopique

Séguéla

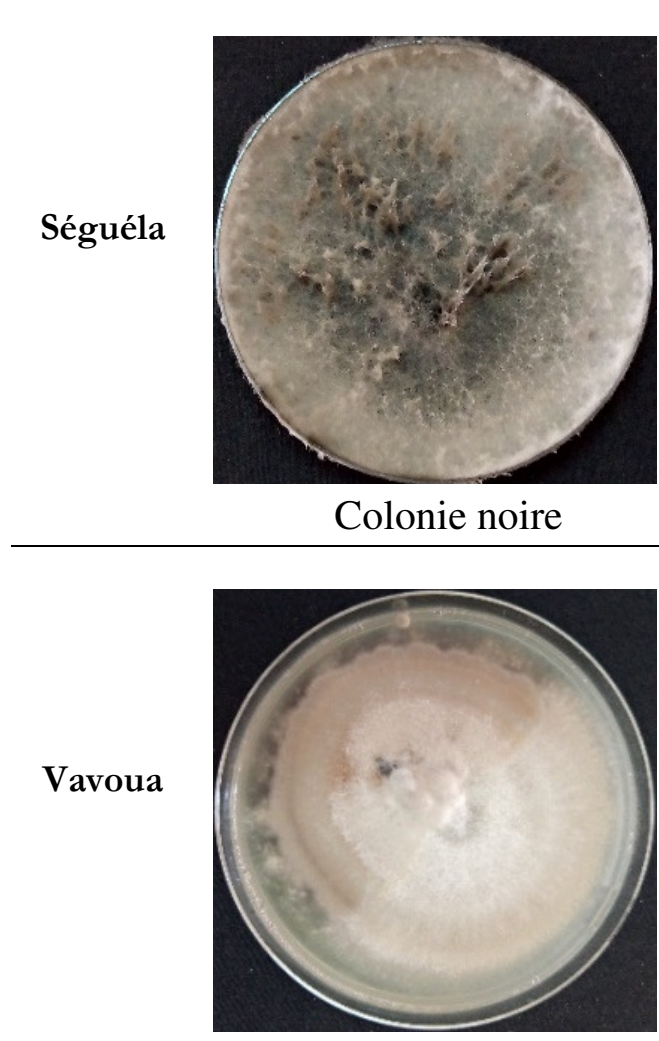

Colonie brune claire
Aspect microscopique

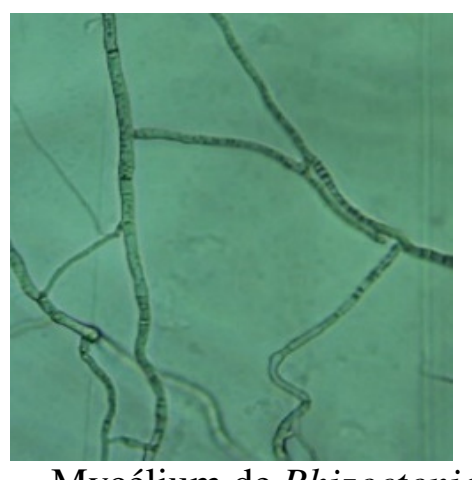

Mycélium de Rhizoctonia sp.

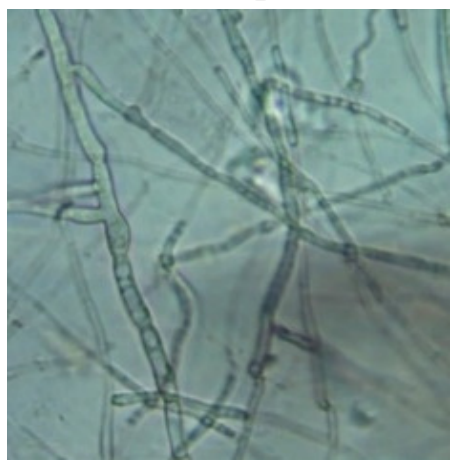

Conidie de NI 1

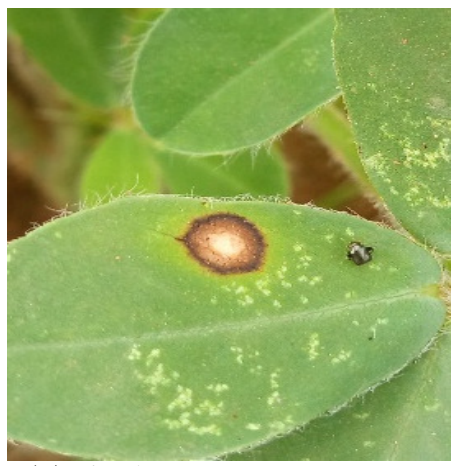

Tache brune avec Intérieur blanchâtre
Séguéla et Vavoua

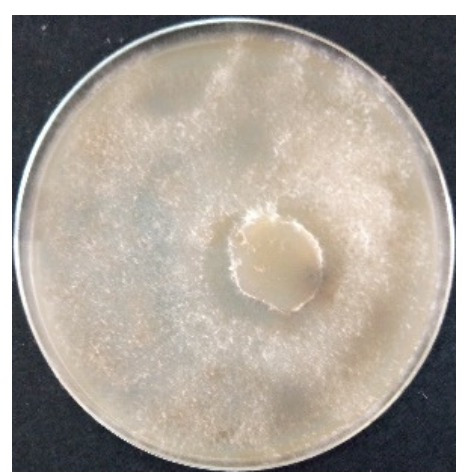

Colonie brune cotonneuse

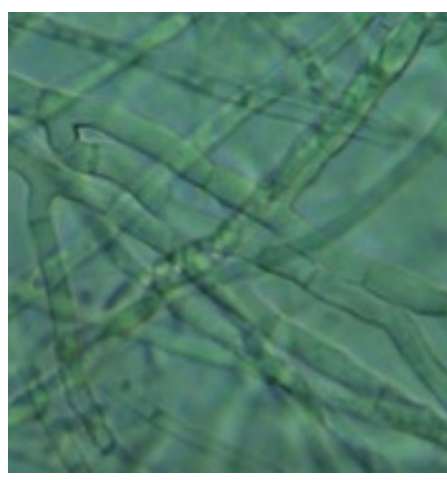

Conidie de Sclérotium sp 
Tableau 3 (Suite) : Caractéristiques macroscopiques et microscopiques des souches fongiques isolées des symptômes communs aux feuilles des accessions provenant de Vavoua de Séguéla

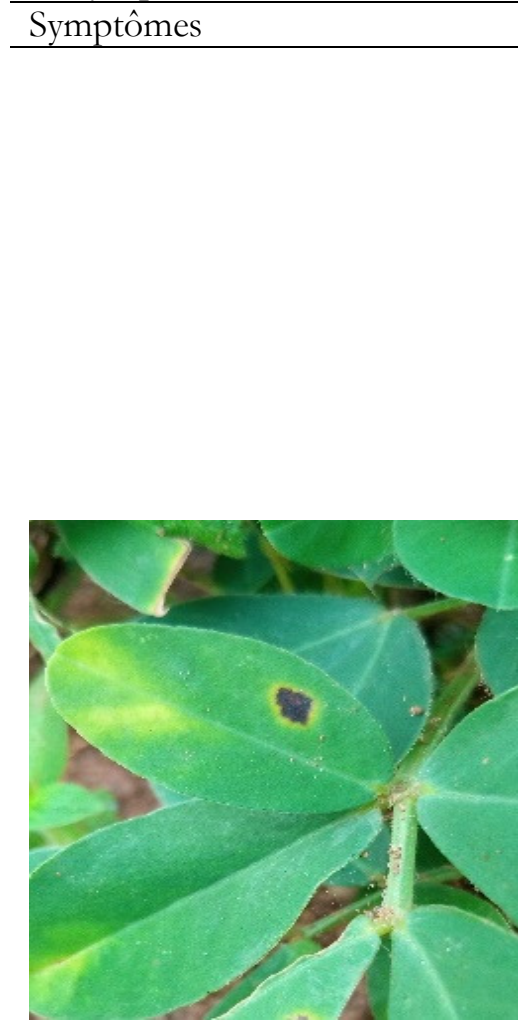

Tache noire entourée d'halo jaune,

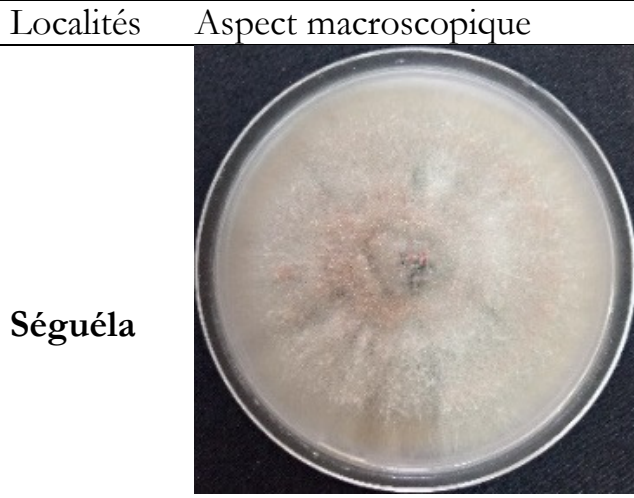

Colonie grise cotonneuse

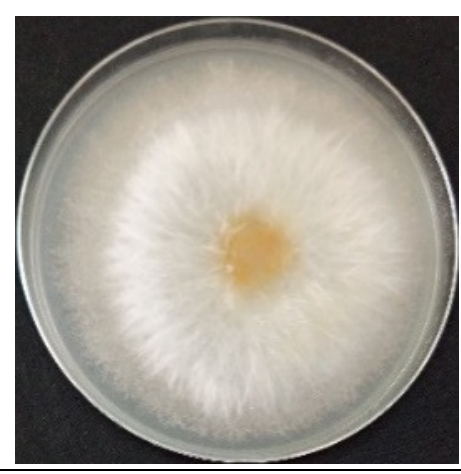

Colonie blanche filamenteuse

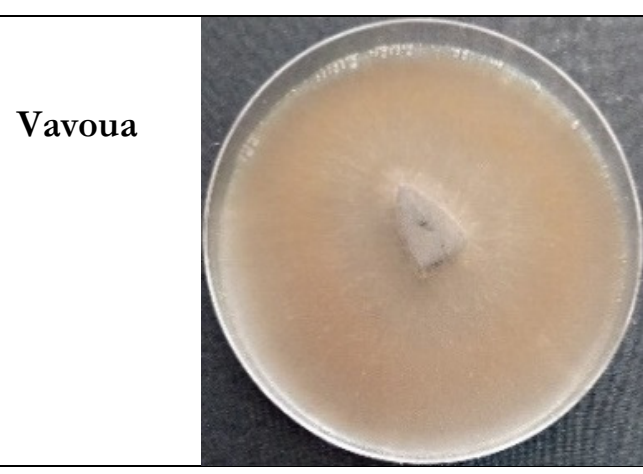

Colonie marron floconneux.

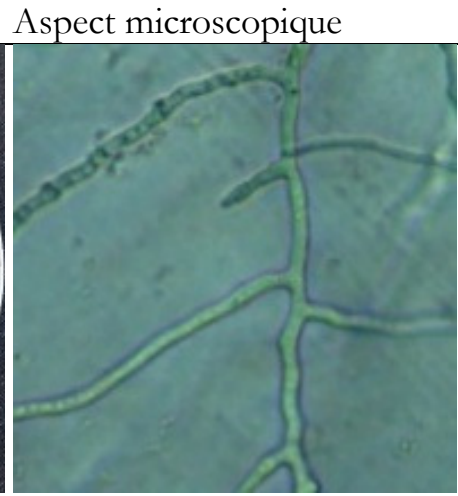

Mycéliums non cloisonnés et

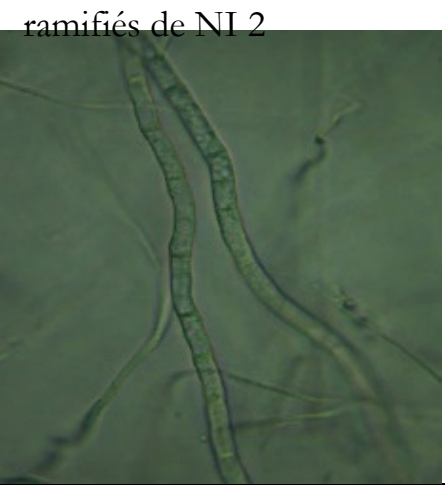

Mycéliums cloisonnés non ramifiés de NI 3

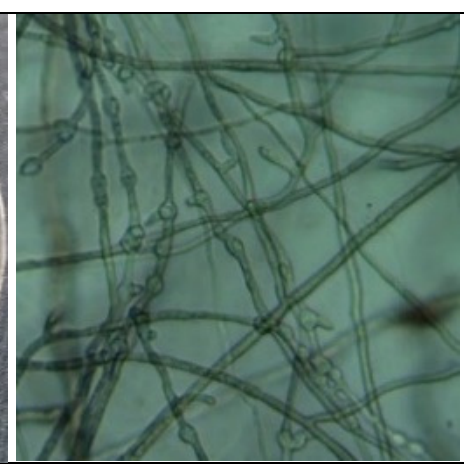

Mycélium cloisonnés et ramifiés de Pytbium sp. 
Tableau 3 (Suite et fin) : Caractéristiques macroscopiques et microscopiques des souches fongiques isolées des symptômes communs aux feuilles des accessions provenant de Vavoua de Séguéla

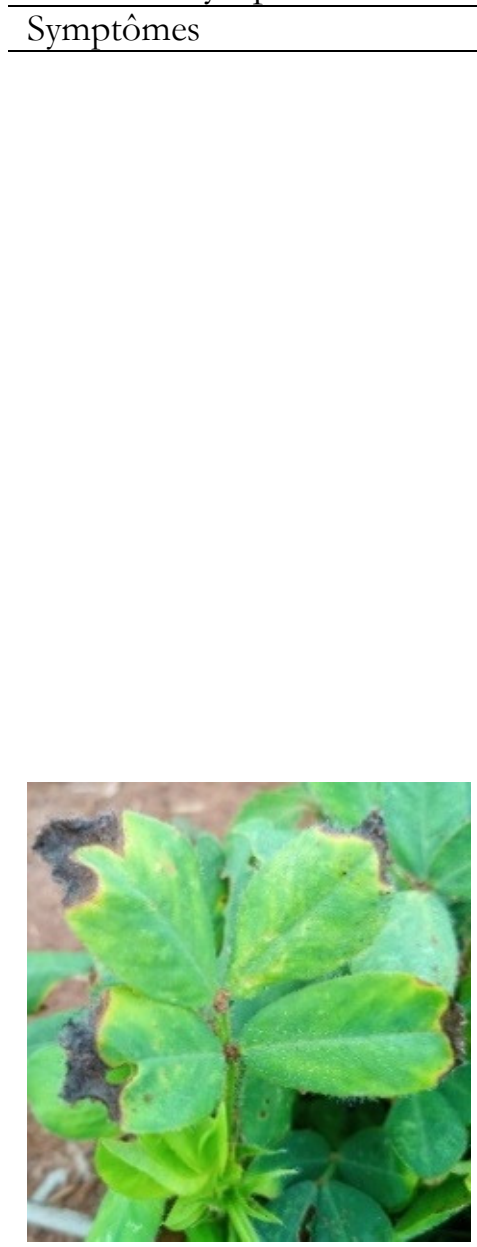

Tache noires sur les bordures des feuilles

Localités

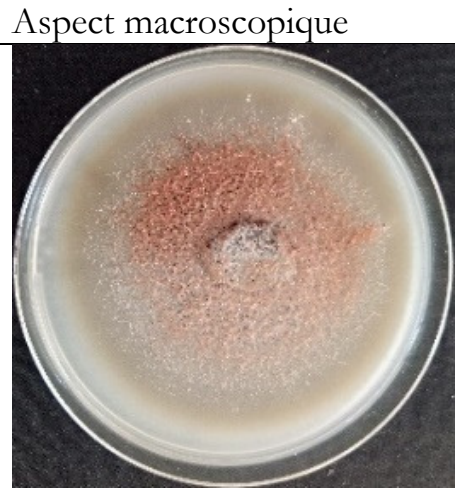

Séguéla

Colonie striée rose blanc

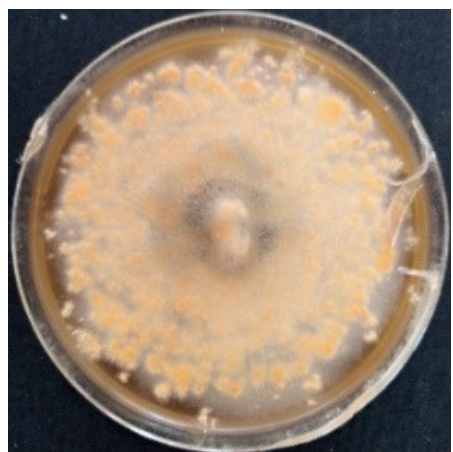

Colonie orangée dispersée

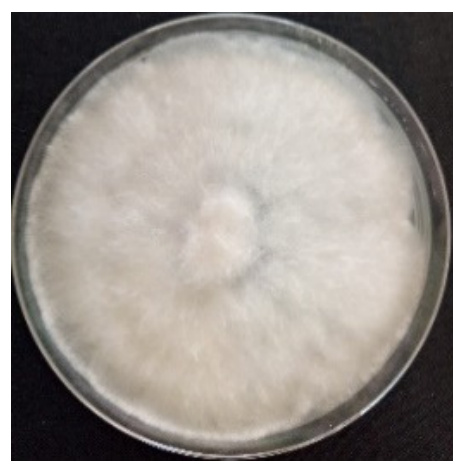

Colonie blanche cotonneuse

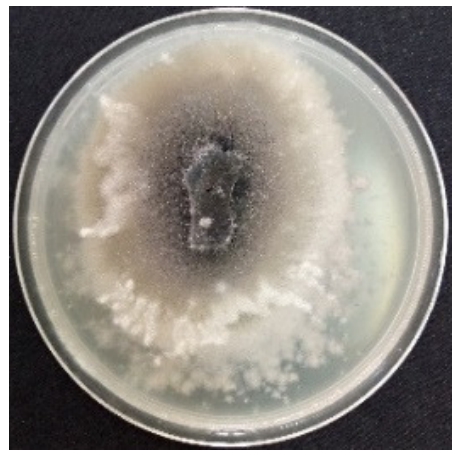

Colonie noire cotonneuse

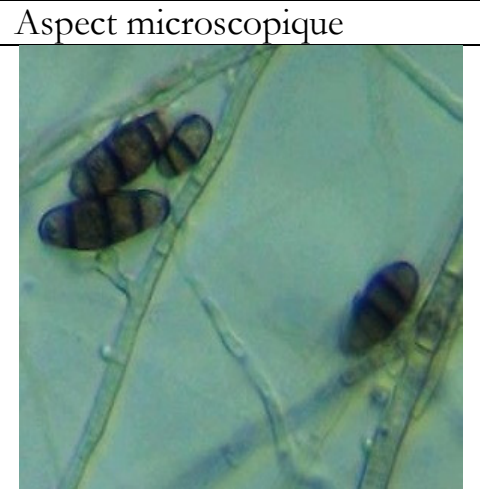

Conidie de Curvularia sp.

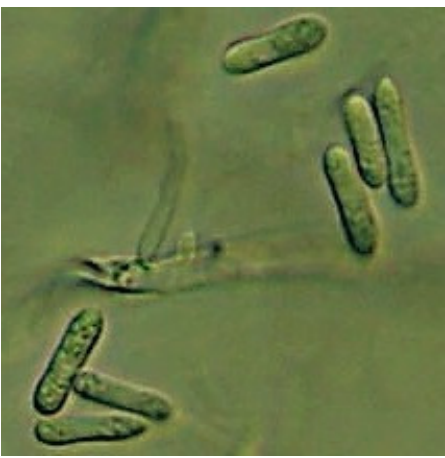

Conidies de Colletotrichum $\mathrm{sp}$

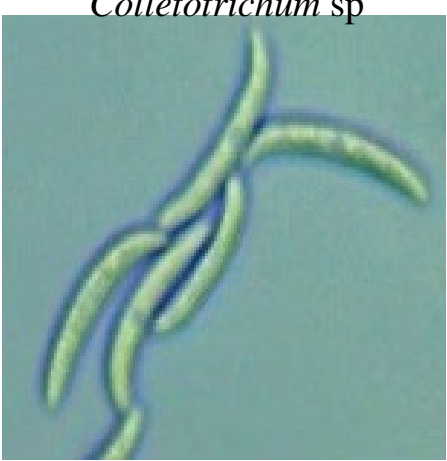

Conidies de Fusarium sp

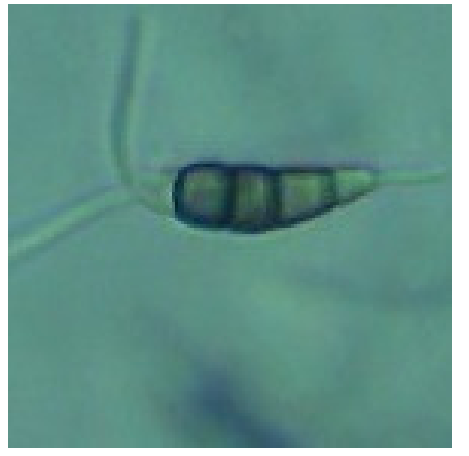

Conidie de Pestalotiopsis sp 
4.2 Symptômes observés et champignons isolés des feuilles d'arachide des accessions de Séguéla : Deux symptômes ont été observés sur les feuilles des accessions de Séguéla. L'un est une tache brune située sur le bord supérieur d'un côté de la nervure principale à la face supérieure de la feuille. De ce symptôme, un seul champignon a été isolé, l'observation macroscopique a montré une colonie cotonneuse de couleur blanche. Ce champignon présente des macrospores en forme de fossiles appartenant au genre Fusarium. L'autre symptôme est caractérisé par de larges taches brunes noirâtres avec un halo jaune de part et d'autre de la nervure principale. Sur le milieu de culture, le champignon isolé de ce symptôme présente une colonie cotonneuse de couleur brune. Au niveau microscopique, le mycélium est ramifié et cloisonné avec des Chlamydospores du genre Colletotrichum (Tableau 4).

4.3 Symptômes observés et champignons isolés des feuilles d'arachide des accessions de Vavoua : Six symptômes ont été observés sur les feuilles des accessions provenant de Vavoua. Le $1^{\text {er }}$ symptôme présente des taches brunes rondes aux contours jaunes sur la face supérieure de la feuille. Le champignon isolé à partir de ce symptôme est de couleur brune cotonneuse sur le milieu de culture. Il présente des mycéliums non cloisonnés avec une tête sporifère du genre Aspergillus.Le $2^{\text {ème }}$ symptôme observé se caractérise par des taches rondes noires sur la face supérieure de la feuille. Sur le milieu PDA le champignon issu de ce symptôme est d'aspect floconneux blanc. L'observation microscopique a montré des conidies grises appartenant au genre Geotrichum. Le $3^{\text {ème }}$ symptôme est caractérisé par des taches brunes sur la face supérieure de la feuille. Le genre Sclerotium a été isolé de ce symptôme et est caractérisé par un aspect blanc filamenteux sur le milieu de culture. $\mathrm{Au}$ niveau microscopique, il a montré des mycéliums cloisonnés et stérile. Le $4^{\text {ème }}$ symptôme est une tache de coloration brune de forme allongée au contour jaune sur la feuille. L'observation sur milieu PDA a présenté une colonie de couleur vert jaunâtre et poudreuse. $\mathrm{Au}$ microscope, les mycéliums non cloisonnés avec une tête sporifère brune de l'espèce Aspergillus sp. a été identifié. Le $5^{\text {ème }}$ symptôme se présente sous forme de taches noires dispersées sur toute la face supérieure de la feuille. Sur le milieu de culture, les colonies du champignon isolé de ce symptôme sont de couleur brune. L'observation microscopique a présenté un mycélium cloisonné dont l'extrémité se présente sous forme de rondelle de couleur sombre. Il s'agit du genre Basipetospora. Le dernier symptôme est caractérisé par de taches rougeâtres parsemées de coloration jaune sur la face supérieure de la feuille. Sur le milieu PDA, le champignon issu de ce symptôme a un aspect cotonneux brun. Au niveau microscopique, il présente des microconidies en forme de chapelet appartenant au genre Griseum (Tableau 5). 
Tableau 4 : Caractéristiques macroscopiques et microscopiques des différentes souches fongiques isolées des symptômes des feuilles des accessions de Séguéla

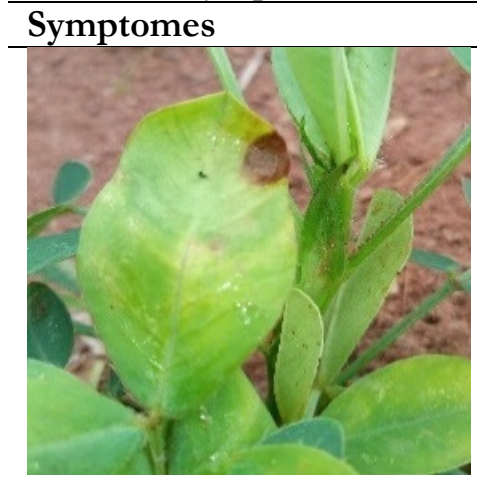

Tache brune en bordure de la feuille

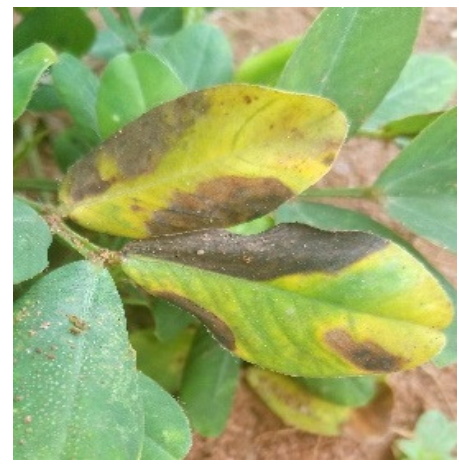

Larges taches brunes noirâtres avec halo jaune

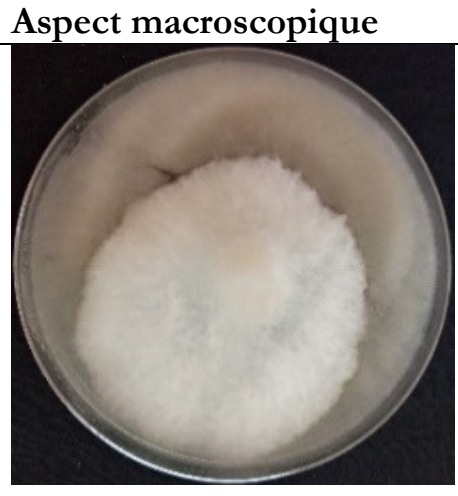

Colonie blanche cotonneuse

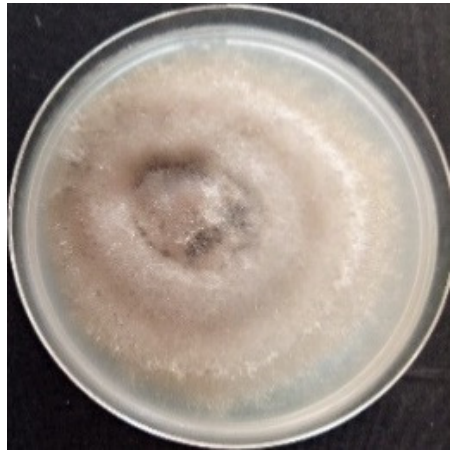

Colonie brune cotonneuse

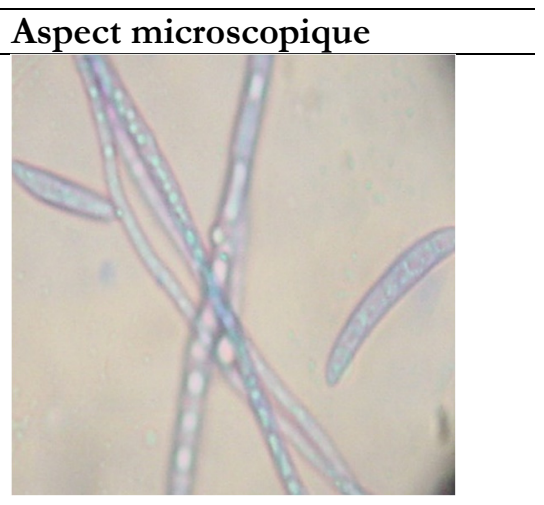

Macrospores de Fusarium sp

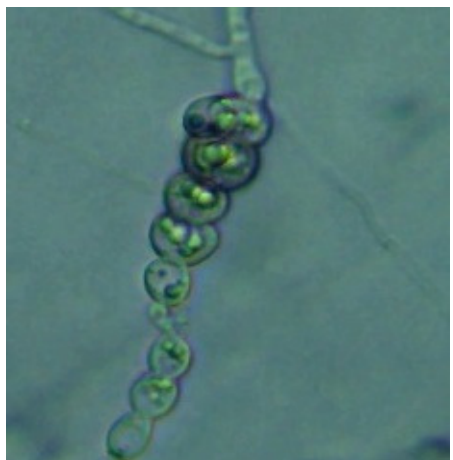

Chlamydospores de Colletotrichum sp. 
Tableau 5 : Caractéristiques macroscopiques et microscopiques des différentes souches fongiques isolées des symptômes des feuilles des accessions de Vavoua

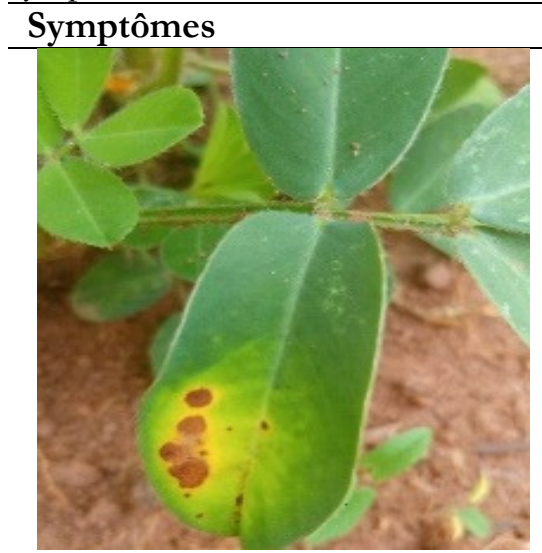

Taches brunes rondes aux contours jaunes

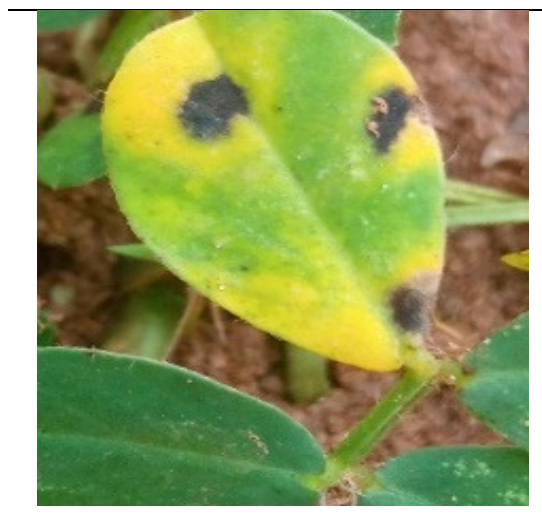

Taches rondes noires

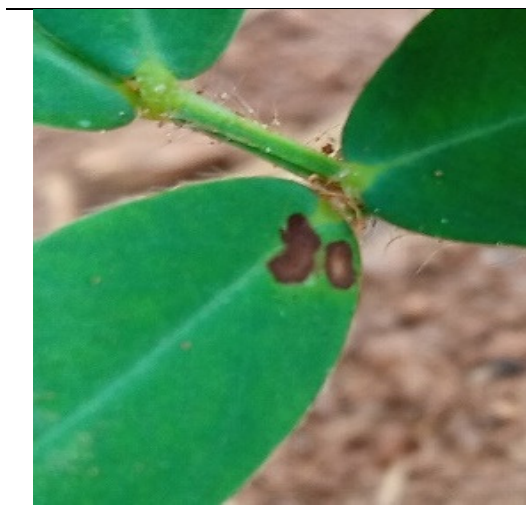

Tache brune

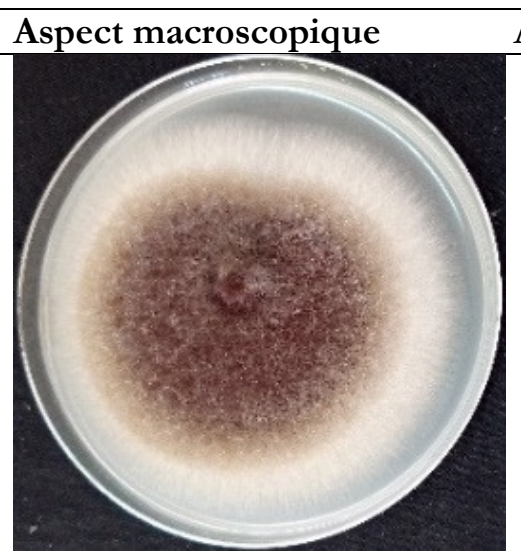

Colonie marron cotonneuse

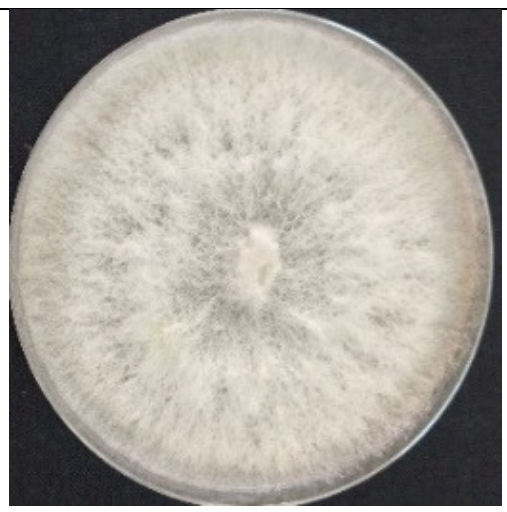

Colonie blanche floconneuse

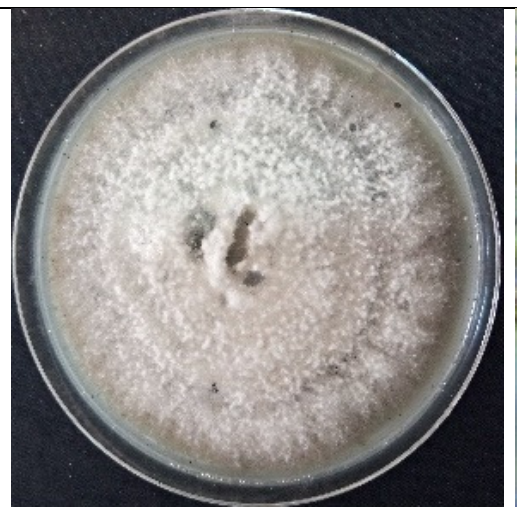

Colonie blanche filamenteuse

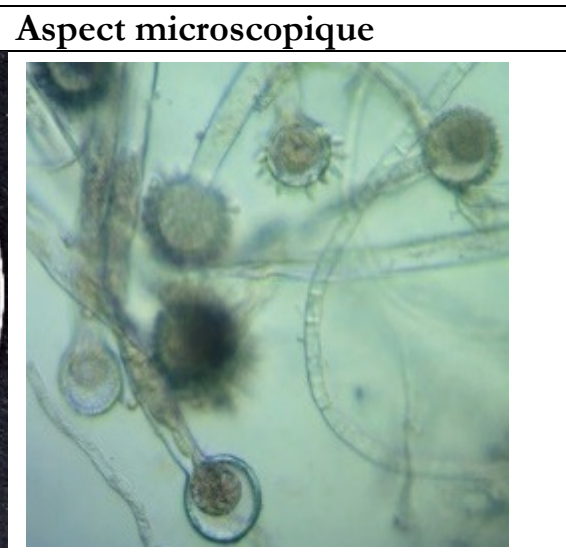

Mycéliums non cloisonnés avec une tête sporifère d'Aspergillus sp 1.

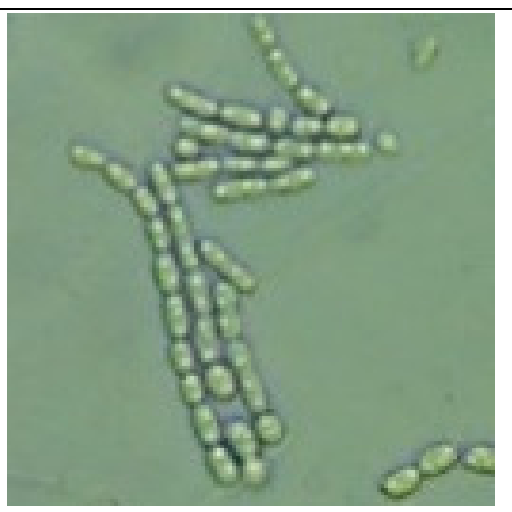

Conidie grise de Geotrichum sp.

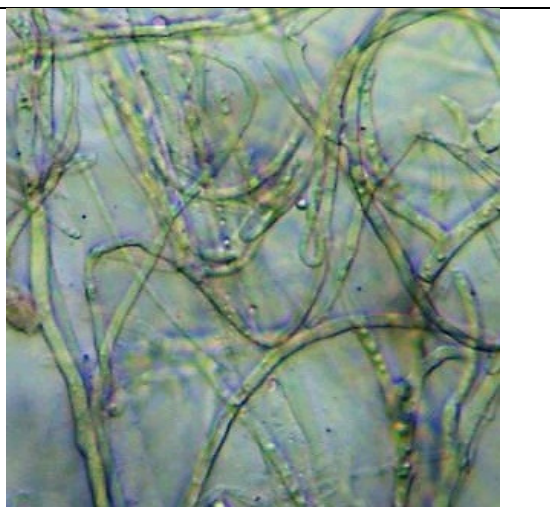

Mycélium de Sclerotium sp. 
Tableau 5 (Suite et fin) : Caractéristiques macroscopiques et microscopiques des différentes souches fongiques isolées des symptômes des feuilles des accessions de Vavoua

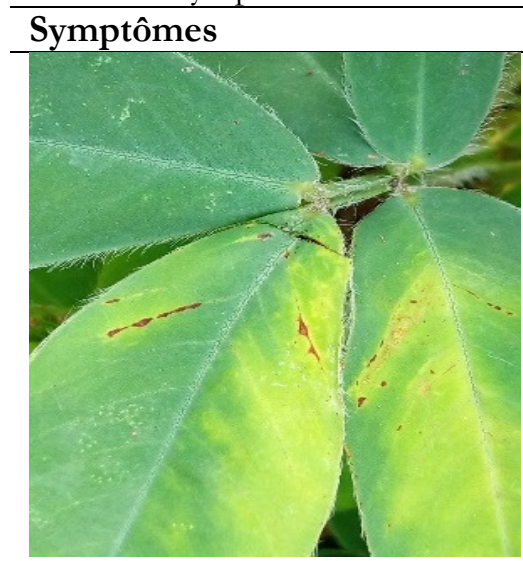

Taches allongées brunes au contour jaune

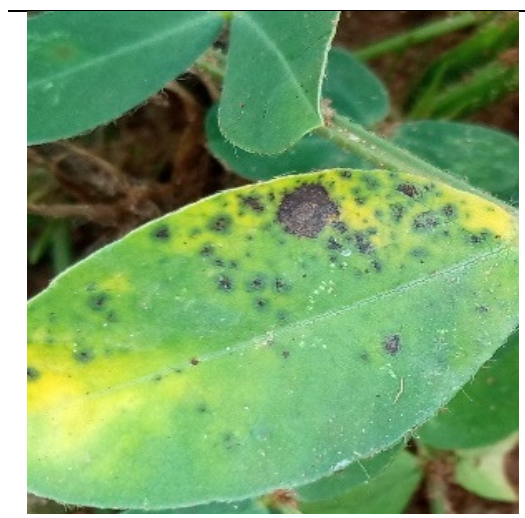

Taches noires dispersées sur la feuille

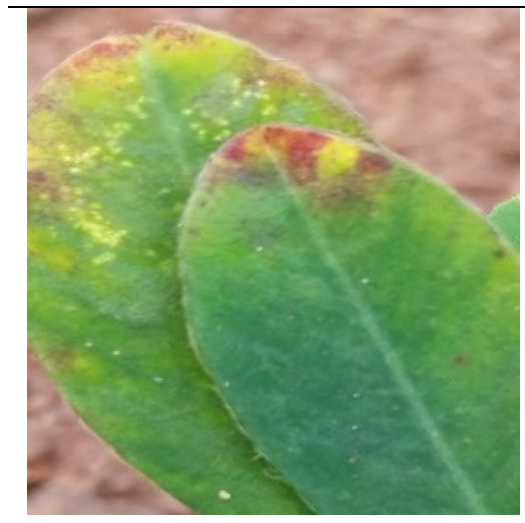

Taches rougeâtres parsemées de coloration jaune

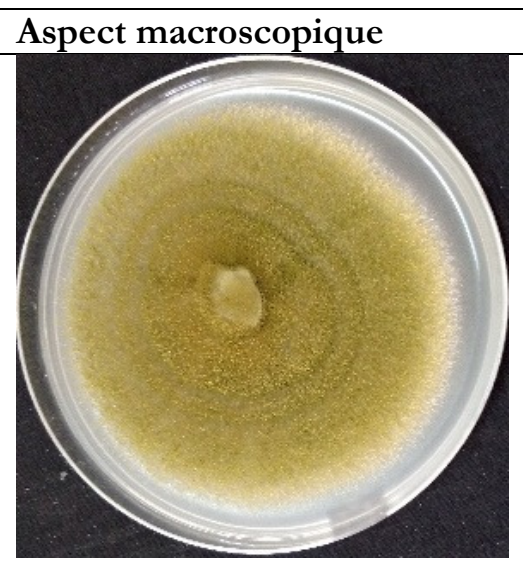

Colonie vert jaunâtre et poudreuse

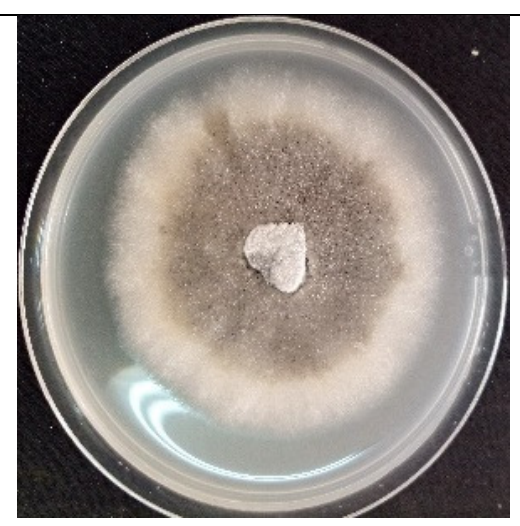

Colonie brune cotonneuse

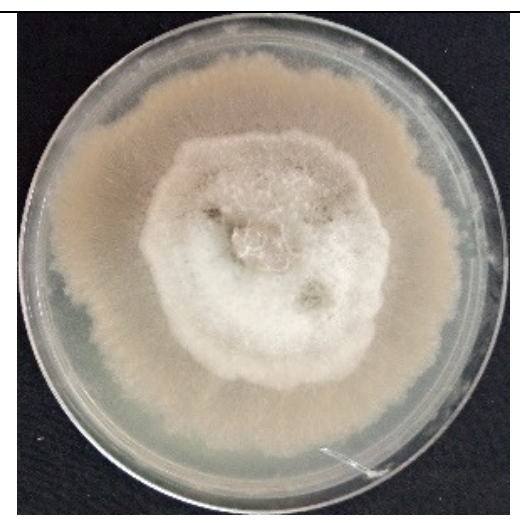

Colonie cotonneuse brune

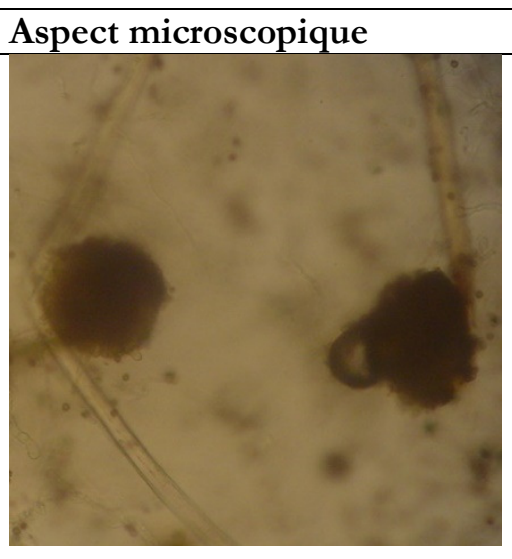

Mycélium et spores d'Aspergillus sp 2.

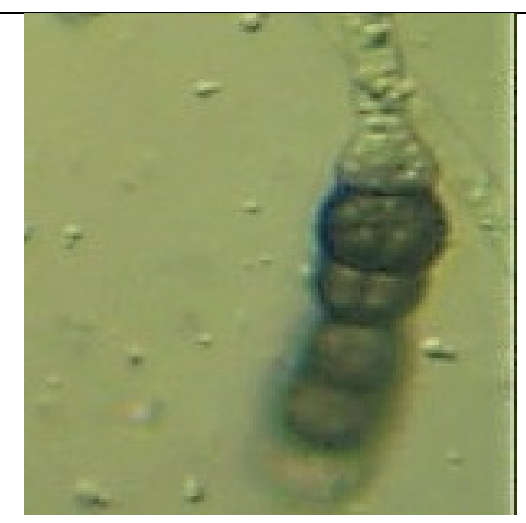

Conidie de Basipetospora sp.

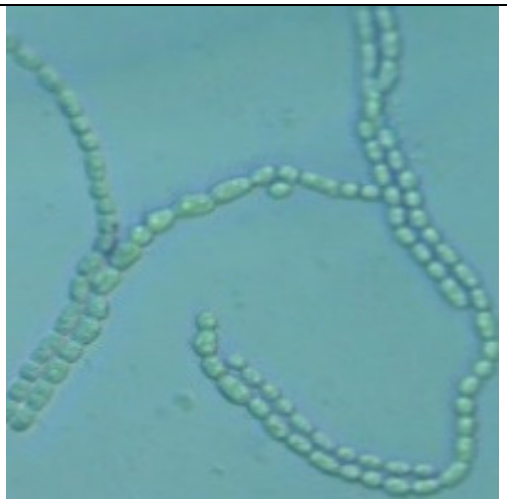

Conidie de Griseum sp. 


\section{DISCUSSION}

Douze symptômes ont été observés sur les feuilles d'arachide. Parmi ces symptômes, quatre ont été à la fois observés sur les accessions des deux localités que sont Vavoua et Séguéla. Par contre, deux autres symptômes ont seulement été observés sur les accessions provenant de Séguéla et six autres uniquement sur les accessions provenant de Vavoua. De ces différents symptômes, dix-neuf isolats fongiques ont été isolés des différents symptômes foliaires de l'arachide. Seize isolats fongiques repartis dans onze genres ont été identifiés. Ce sont Aspergillus, Basipetospora, Colletotrichum, Curvularia, Fusarium, Geotrichum, Griseum Rhizoctonia, Pestalotiopsis, Pythium, Sclerotium. Les trois isolats restants, n'ont pu être identifiés et n'ont par conséquent été associés à aucun genre fongique. La variabilité de symptômes observée sur les feuilles des deux localités pourrait être due aux mêmes agents pathogènes. Cette variabilité pourrait aussi être due aux relations que pourraient avoir les plantes d'arachide avec les écosystèmes environnants. En effet, l'expérience ayant été menée en milieu naturel et contiguë à d'autres cultures, les contaminations pourraient être dues à l'action de la pluie ou du vent qui pourraient transporter des spores de champignon sur de longues distances. Aussi, le développement d'un symptôme sur les feuilles d'arachide pourrait impliquer la présence d'une ou plusieurs sources d'infection, des hôtes sensibles et des conditions favorables à son évolution. Ces observations sont en cohérence avec ceux d'Anahosur (1992) qui a indiqué qu'au niveau des cultures, les champignons pourraient se développer sur la matière organique en décomposition ou non. De plus, un seul parasite

\section{CONCLUSION}

La présente étude a monté qu'une diversité de symptômes foliaires est associée à la culture de l'arachide. L'isolement et l'identification de souches fongiques associées aux feuilles symptomatiques d'Arachis hypogaea en culture a permis l'obtention d'une diversité de champignons. Les champignons isolés, ont variés en fonction des accessions en provenance peut également induire différents symptômes foliaires selon les facteurs qui entourent l'apparition de la maladie. Selon Howell (2003) les lésions ne sont pas colonisées seulement par les pathogènes, mais également par les saprophytes. Mouria et al. (2003) corroborent qu'une lésion peut être initiée par un pathogène facultatif et colonisée par d'autres. Aussi, différents agents pathogènes facultatifs peuvent induire des symptômes similaires. Différents genres fongiques ont été isolés d'un même symptôme ainsi qu'un même genre fongique a été isolé sur au moins deux symptômes différents. Ces résultats sont en accord avec ceux de Koffi (2015), qui affirme que dans les conditions naturelles, d'autres champignons s'installent à la suite de l'agent principal. Certains champignons participent à la destruction des tissus soit en poursuivant l'action de Colletotrichum, soit en envahissant les organes affaiblis indirectement par la maladie. C'est le cas de Trichoderma dont certaines espèces seraient apparemment des antagonistes importants de certains genres comme Fusarium. En somme, la présence de différents genres fongiques associés à un symptôme pourrait montrer qu'il n'y a pas de spécificité de genre fongique et de type de symptômes décrit. De plus, Assanvo et al. (2002) et N'zue et al. (2008) ont montrés qu'une diversité de genres fongiques attaque le manioc en Côte d'Ivoire. Cette diversité fongique serait due selon les résultats des travaux de d'Howell (2003) et de Mouria et al. (2003) au fait qu'une association de genres fongiques dans la plante est due à l'entrée de ces agents par une ouverture initiée soit naturellement, par un outil ou par un autre agent phytopathogène.

d'une localité à une autre. Des dix-neuf souches obtenues, seize (16) ont été identifiées et reparties dans onze genres fongiques. Ce sont, les genres Aspergillus, Basipetospora, Colletotrichum, Curvularia, Fusarium, Geotrichum, Griseum Rhizoctonia, Pestalotiopsis, Pythium, Sclerotium. Trois souches non identifiés ont été aussi observés. $\mathrm{Vu}$ la variabilité de ces souches et les nombreux 
problèmes rencontrés lors de la culture d'arachide et sachant que les symptômes n'étaient pas spécifiques aux genres de champignon rencontrés sur les feuilles, l'identification peut avoir souffert d'une certaine

\section{REMERCIEMENTS}

Nos remerciements vont à l' endroit des autorités de l'Université Jean Lorougnon Guédé de Daloa (Côte d'Ivoire) et du groupe de

\section{REFERENCES}

ANADER: 2009. La production vivrière: un enjeu national. Bulletin de liaison de l'agence nationale de développement rural. Bull. Liaison. 12 p.

Anahosur KH: 1992. Sorghum and millets diseases: A second world review, In: AP Patancheru (Eds), International crops research institute for the semi-arid tropics, India: pp. 45-56.

Assanvo JB, Agbo GN, Behi YEN and Coulin PZ: 2002. La microflore du ferment de manioc pour la production de l'attiéké adjoukrou à Dabou (Côte d'Ivoire). Revue Internationale des Sciences de la Vie et de la Terre $\mathrm{N}^{\circ}$ spécial : 286-299.

Barnett HL. and Hunter BB: 1998. Illustrated genera of imperfecti fungi. Third edition Burgess Publishing Company. Minneapolis, $241 \mathrm{p}$.

Boli ZA, Zoue LT, Alloue-Boraud WM, Kakou CA. and Koffi N R: 2013. Proximate composition and mycological characterization of peanut butter sold in retail markets of Abidjan (Côte d'Ivoire), Journal Application of Bioscience 72: 58225829.

Botton B, Breton A, Fevere M, Gauthier S, Guy P h, Larpent JP, Reymond P, Sanglier JJ, Vayssier Y. and Veau, P: 1990. Moisissures Utiles et Nuisibles, Importance industrielle, Paris: Massons $1248 \mathrm{p}$.

Boussaber E, Kadmiri IM, Hilali L. et Hilali A: 2012. Isolement des souches d'actinomycètes productrices de inexactitude. Par conséquent, d'autres études, y compris des tests de pathogénicité, sont nécessaires pour établir un lien plus précis entre les champignons et les maladies d'origine fongique de l'arachide.

recherche de physiologie végétale de cette Université pour leur contribution à la réalisation de ce travail.

substances antifongique. ScienceLib Editions Mersenn. 4: 2111-4706.

Christie ME, Kyamureku P. and Kaaya A: 2015. Farmers, peanuts, and aflatoxins in Uganda: A gendered approche Development Practic 25(1): 4-18.

Diakité A, Gouli Bi IM, N'dri DK. and Yapo J: 2017. Détermination de la contamination par l'aflatoxine B1 de la pâte d'arachide consommée par la population en Côte d'Ivoire: Intérêt de la chromatographie sur couche mince, International Journal of Biological Chemical Science 11(4): 1646-1654.

Dzomeku IK, Abudulai M, Brandenburg RL. and Jordan DL: 2009. Survey of Weeds and Management Practices in Peanut (Arachis bypogaea L.) in the Savanna Ecology of Ghana, Peanut Science 36: 165173.

FAOSTAT: 2013. La base de données statistique de l'organisation des nations unies pour l'alimentation et l'agriculture. fao.org, 59 p.

Guchi E, Ayalew A, Dejene M, Ketema M, Asalf B. and Fininsa C: 2014. Occurrence of Aspergillus Species in Groundnut (Arachis bypogaea L.) along the Value Chain in Different Agro-Ecological Zones of Eastern Ethiopia, Journal of Applied and Environnemental Microbiology 2(6): 309-317.

Guchi E: 2015. Effect of Storage Time on Occurrence of Aspergillus species in Groundnut (Arachis bypogaea L.) in Eastern Ethiopia, Journal of Applied and Environmental Microbiology 3(1): 1-5. 
Howell C: 2003. Mechanisms employed by Trichoderma species in the biological control of plant diseases: the History and Evolution of Current Concepts, Plant Disease 87(1): 4-10.

Koffi AMH: 2015. Pathogénicité et diversité génétique des parasites fongiques des graines et feuilles de Lagenaria siceraria (Molina) Standl (Curbitaceae) de cinq localités (Alépé, Bondoukou, Bongouanou, Divo et Korhogo) de la Côte d'Ivoire, Thèse unique, Université Nangui Abrogoua, Abidjan, Côte d'Ivoire, $169 \mathrm{p}$.

Manfred M. et Nicole M: 2000. Précis des risques alimentaires. Tec et Doc. édition, Londres-Paris-New-York. 442 p.

Morgavi DP. and Riley RT:2007. An historical overview of field disease outbreaks known or suspected to be caused by consumption of feeds contaminated with Fusarium toxins. Animal Feed Science Technology 137: 201-212.

Mouria A, Ouazzani TA. et Douira A: 2003. Etude de certains facteurs favorisant le Maintien de l'activité antagoniste de Trichoderma harzianum à l'égard de Helminthosporium oryzae sur les feuilles de riz, Sciences et Techniques 5: 50-66.

Ndjeunga J, Ntare BR, Waliyar F. and Ramouch M: 2006. Groundnut seed systems in West Africa. International Crops Research Institute for the Semi-Arid Tropics $232 \mathrm{p}$.

Noba K, Ngom A, Guèye M, Bassène C, Kane M, Diop I, Ndoye F, Mbaye MS, Kane A. and Tidiane Ba A: 2014. L'arachide au Sénégal: Etat des lieux, contraints et perspectives pour la relance de la filière, Oilseeds and fats Crops and Lipids 21(2): 15.

N'Zue B, Zobouri GP, Yapi GV. and Djédji C: 2008. Bien cultiver le manioc en Côte d'Ivoire, Fiche technique, Centre National de recherche Agronomique, Abidjan, 50 p.

Ouattara SPB, Nikiema PA. and Traore AS: 2011. Caractérisation de souches
d'Aspergillus spp isolées des graines d'arachides cultivées au Burkina Faso, Afrique de l'Ouest. International Journal of Biological and Chemical Sciences 5(3): 12321249.

Reddy ECS, Sudhakar C. and Reddy NPE: 2011. Aflatoxin contamination in groundnut induced by Aspergillus flavus type fungi: A critical review, International Journal of Applied Biology and Pharmaceutical Technology 2(2): 180-192.

Sharma V, Purohit GR, Arya RS. and Harsh M: 2006. Evaluation of some complete rations in sheep incorporating unconventional feed resources of arid zone of Indian, Animal Nutrition and Feed Technology 6(1): 135-141.

Yussif IJ, Charles K, Mahama O, Kwabena A. and Yirzagla J: 2014. Farmers' Perception and Farming Practices on the Effect of Early and Late Leaf Spots on Groundnut Production in Northern Ghana, Journal of Biological and Agricultural Healthcare 4(19): 22-28. 